\title{
COMUNIDADES VIRTUALES EN EL CIBERESPACIO
}

\author{
FRANCISGO JAVIER VALIENTE \\ InVestigador del Departamento de Periodismo. Universidad San Pablo-CeU de Madrid
}

$\underline{\text { Resumen }}$

Las nuevas tecnologías de la comunicación están desarrollando nuevas formas de socialización. Alrededor de Internet han ido apareciendo comunidades virtuales que desarrollan sus propias formas de funcionamiento. En el artículo, partiendo de la definición de los términos comunidad y virtual, se señalan las características de este nuevo tipo de socialización y el papel que muchas de estas comunidades pueden jugar en la formación de lo que Pierre lévy denomina la inteligencia colectiva. Después de presentar una clasificación de las comunidades virtuales, se analizan algunos aspectos que suelen concitar más críticas sobre las relaciones que en estas comunidades se crean como el aislamiento de los individuos, el problema de la identidad y el carácter efímero o la precariedad de las relaciones que en ellas se establecen.

Palabras clave

Comunidades virtuales, socialización mediática, nuevos tipos de socialización.

\section{Abstract}

The new communication technologies are spawning new forms of socialisation. A cluster of virtual communities has gradually built up around internet, each developing its own modus operandi. This article, working from the definition of the terms community and virtual, portrays the characteristics of this new type of socialisation and the role that many of these communities might play in the building up of what Pierre calls "collective intelligence". First of all it offers a classification of these virtual communities, after which it analyses some of the factors that usually excite most criticism over the relations created in these communities, such as the isolation of individuals, the problem of identity and the ephemeral and superficial character of the relations established therein.

Key Words

Virtual communities, media socialisation, new types of socialisation. 


\section{INTRODUCCIÓN}

Muchas de las funciones de la vida ordinaria tienen un doble virtual, especialmente las referidas a las relaciones con los otros, al conocimiento, la información y el compartir intereses. La Red no es sólo un lugar por donde transitar, por donde ir de paso; es también un lugar donde habitar. En Internet se están creando comunidades que utilizan la Red como medio de agregación. Comunidades virtuales entendidas como "conjunto de personas que, intercambiándose mensajes de correo electrónico, charlando simultáneamente o participando en juegos de rol, desarrollan lazos más - menos estables y duraderos fundados sobre intereses comunes" (Cipolla-Dell'Aquilla, 1998). Internet es un medio de comunicación que, a diferencia de los medios anteriores, está convirtiéndose en lugar de socialización. Este es el aspecto que quiero abordar en este trabajo.

Pero, ¿qué entendemos por comunidades virtuales?, ¿̇cómo funcionan?, ¿̇a qué nos referimos cuando hablamos de ellas?

\section{COMPRENDER LAS COMUNIDADES VIRTUALES}

Parece que al hablar de comunidades virtuales estamos enfrentando dos términos contrapuestos. El sustantivo "comunidad" habla de consistencia, de existencia real, de un tipo de relación concreta entre personas en un espacio físico determinado. El adjetivo "virtual", en cambio, se suele tomar como opuesto a real, y se entiende como falso e ilusorio, como no existente. Parece contradictorio que pueda existir, en este sentido, una comunidad de personas que sea, al mismo tiempo, virtual. Tal vez la contradicción provenga de haber tomado el término de la realidad virtual, que es un tipo de realidad artificial' generada por programas informáticos; un tipo de realidad que sólo existe mientras está funcionando el programa. Un tipo de realidad que existe en la medida en que están activos los ordenadores que la producen y sólo existe dentro de ellos. Usando dispositivos especiales (cascos, visores, guantes), el hombre puede llegar a interactuar con ella; puede tener la sensación de manipularla.

Pero virtual no se entiende, en este contexto, como irreal, falso o ilusorio. No se trata de un sustituto de lo real, sino como una forma de actualizarlo, como una posibilidad más de lo real (Lévy, 2001: 88); en este caso como una forma distinta de comunidad. Virtual, aquí, se entiende más como la tecnología de la comunicación que se utiliza para ponerse en relación con los otros. Al hablar de las comunidades virtuales, no se habla de la sustitución de las comunidades tradicionales que se organizan fuera del ciberespacio. La clave no está en el adjetivo, sino en el sustantivo.

Lo que se quiere poner de manifiesto utilizando el concepto 'virtual', es que existen nuevos soportes técnicos para relacionarse con los demás. 'Virtual' se utiliza para referirse a la tecnología

\footnotetext{
' "Espacios metafóricos que emergen solamente a través de la interacción con el ordenador, donde la gente navega con un hardware particular" (Turke, 1997: 268).
} 
utilizada (ordenador, software de conexión a la Red y aplicaciones apropiadas), y que permiten un nuevo tipo de socialización diferente "pero no por ello inferior a las formas anteriores de interacción social" (Castells, 2001: 146).

Pero es que no sólo resulta equívoco el término 'virtual'. También el término 'comunidad' se utiliza para designar realidades diversas y referirse a distintos tipos de agregación de personas.

\section{DEFINICIÓN Y TIPOS DE COMUNIDAD}

Por comunidad, en sentido amplio, entendemos un conjunto de personas unidos por factores de diversa índole (lengua, etnia, religión, territorio,...) que les llevan a relacionarse e interactuar entre ellos más que con miembros de otros grupos (Ferri, 1999: 78). Hablando de comunidad, se puede definir teniendo en cuenta dos aspectos principales (Squillacciotti, 2001: 296). Por un lado, entender 'comunidad' como la red de relaciones interpersonales que se establecen entre los individuos. Por otra parte, hacer hincapié en el aspecto espacial y subrayar, del término 'comunidad', la relación entre los individuos que ocupan un territorio determinado con unos mismos objetivos.

Características importantes que definen a una comunidad, según el antropólogo de la cultura Ugo Fabietti (Fabietti, 1999: 47), son el sentido de pertenencia, de exclusión y de identidad y, dependiendo del contexto y de connotaciones diferentes que esas características pueden adquirir, las comunidades serán diversas. En la comunidad hay una voluntad natural de mantener unas relaciones sociales, como en el caso de los grupos parentales donde se dan relaciones interpersonales que "permiten una comunidad de sentimientos, emociones e ideas"2, frente a una voluntad racional que se verifica en las sociedades, entendiendo éstas como conjunto de grupos o individuos que colaboran en la consecución de fines prefijados.

Fabietti (Fabietti, 1999: 43-60) distingue, a lo largo de la historia, dos grandes tipos de comunidad con un tipo característico de memoria, pues para este autor es la memoria lo que explica la existencia y cohesión de las comunidades. Un primer tipo de comunidad sería la que él denomina de sociabilidad densa que correspondería a grupos en los que existe una relación estrecha, de consanguinidad en sentido amplio, afectiva entre los miembros. El tipo de memoria es genealógica, generalmente una construcción con bastantes elementos ficticios que sirve de relato fundador de esa comunidad. Con la aparición de los estados modernos, aparece otro tipo de comunidad, la comunidad imaginada que se basa sobre la idea de nación que es también una ficción, en el sentido que es una selección de algunos aspectos (lengua, una cultura, orígenes territoriales, etc.) que sirven para constituir un grupo homogéneo sobre el que apoyar la idea de nación. La memoria, en este tipo de comunidad, es una memoria nacional, muchas veces étnica, que subraya algunos acontecimientos y oculta otros, que filtra sucesos de la historia y los lee en una clave que sirvan para sustentar la idea de nación.

\footnotetext{
2 La distinción entre comunidad y sociedad está tomada del sociólogo Ferdinad Tönnies citado por FERRI, La rivoluzione digitale, 78.
} 
Las nuevas tecnologías han permitido el nacimiento de un nuevo modo de formar comunidad: las comunidades telemáticas que encuentran su lugar de existencia en Internet. Comunidades a las que pertenecen personas de lengua, nacionalidad, cultura diversas, y que sin embargo se reconocen como pertenecientes a esa comunidad. Por la naturaleza de los medios utilizados, la memoria es, en este caso, una memoria sin espacio ni tiempo; el espacio es anulado pues lo que cuenta es la posibilidad de conexión que depende de la velocidad y que determina el tiempo.

En este nuevo espacio de socialización, en este nuevo mundo, los individuos "deben reformular la propia identidad y la idea de la propia pertenencia" (Fabietti, 1999: 57). Esas comunidades pueden ser creadas ex novo dentro de la Red, o ser continuación de las ya existentes. Las comunidades virtuales, como los otros tipos de comunidades, parten de una ficción, los miembros comparten algunos rasgos comunes, tienen un tipo de memoria específico, un territorio donde desarrollarse -en este caso el no-lugar de la Red-y un tipo de identidad y sentido de pertenencia.

Como ante el fenómeno Internet en general, también ante la existencia de las comunidades virtuales existen posturas encontradas. Para algunos autores (Maldonado, Talbot) ${ }^{3}$, las comunidades virtuales representan formas comunitarias débiles, sin la suficiente fuerza de cohesión como para considerarlas verdaderas comunidades. Otros (Rheingold, Turkle) consideran las comunidades virtuales como una nueva forma de comunidad, una evolución de las comunidades ya existentes y, aunque diversas de estas, con características similares a las comunidades tradicionales. Presentar las dos posturas, nos ayuda a captar de forma esquemática los aspectos positivos y los límites de este fenómeno.

\section{UN NUEVO TIPO DE SOCIALIZACIÓN}

Uno de los primeros estudios sobre las comunidades virtuales fue el realizado por Howard Rheingold en 1994, en el que describe el funcionamiento de la comunidad WELL (Whole Earth Electronic Link), fundada en 1985 por S. Brand y L. Brilliant, y de la que él es un entusiasta promotor. Esta comunidad, que todavía hoy es operativa (www.well.com) ${ }^{4}$, consta de numerosos grupos divididos por ámbitos de interés, con grupos de discusión sobre temas que proponen los mismos miem-

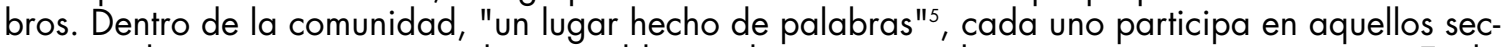
tores en los que está interesado y establece relaciones con las personas que encuentra. En la comunidad los miembros disponen de espacio para construir su propia página web, inscribirse en listas de correo, participar en debates, acceder a servicios de documentación, etc.

\footnotetext{
3 T. Maldonado y S.L. Talbot, entre los primeros, H. Rheingold e S. Turkle entre los segundos (Carbone - Ferri, 1999: 13).

4 Para pertenecer a ella es necesario pagar 15 ó 10 dólares mediante tarjeta de crédito, según el tipo de cuenta que se quiera tener, aceptar las normas de funcionamiento de la comunidad y rellenar un formulario de datos personales. Además se pide, a cada nuevo miembro, hacer una presentación personal (bio) que será lo que los otros miembros conocerán del individuo de forma directa.

${ }_{5}$ Así se define la misma comunidad en su página de presentación, www.well.com/aboutwell.html.
} 
Para Rheingold, el paso a este tipo de comunidad que se está viviendo en nuestra época, encuentra un parangón en el paso que se produjo en la Europa rural y precapitalista, a la Europa de las ciudades y moderna. Igual que entonces se creó un nuevo tipo de sociedad, de comunidad, ahora estaría naciendo una nueva forma de agrupación en un nuevo espacio de comunidad (Rheingold, 1994: 74). Gracias a las nuevas tecnologías, en estas comunidades puede establecerse un contacto directo con los demás miembros de la sociedad, un tipo de contacto más flexible y creativo que en la sociedad industrializada moderna. Rheingold, entusiasta de estas nuevas comunidades, no pone de manifiesto los aspectos negativos o problemáticos de estas comunidades ni, como señala Paolo Ferri (Ferri, 1999b: 76), el carácter minoritario de este tipo de comunidad. Este es un elemento que tener en cuenta para situarlas en su justo lugar.

Una posición crítica ante este tipo de comunidades, entre otras, es la que representa Tomás Maldonado (Maldonado, 1997) quien pone de manifiesto la precariedad de las relaciones en las comunidades virtuales. Para este autor estas comunidades son formas de interacción débiles, pues se basan sólo en compartir intereses comunes. Las personas que forman parte de ellas lo hacen porque comparten unas mismas ideas y valores. En estas comunidades hay muy poco espacio para el conflicto. Precisamente el conflicto, la confrontación, y la forma de cómo se asimila y gestiona el conflicto en una comunidad, permite una verdadera interacción comunitaria. Además, se señala la escasa influencia de estas comunidades en la sociedad.

De nuevo, apocalípticos e integrados ante esta realidad. Los pioneros de las comunidades virtuales veían la solución a los problemas de socialización; la utopía de una socialización generalizada se podía hacer realidad. Sus detractores veían otra forma de escape de la realidad y de individualismo exagerado. El desarrollo de las comunidades virtuales ha ido atemperando las posiciones y frente a las afirmaciones de que Internet es una fuente de comunidad renovada $o$, al contrario, de que constituye una causa de alineación y escape del mundo real, parece ser que la interacción social en la red, en general, no tiene un efecto directo sobre la configuración de la vida cotidiana, más allá de añadir la interacción on line a las relaciones previamente existentes (Castells, 2001: 140).

Lo cierto es que se están estableciendo nuevas formas de agrupación que utilizan la tecnología de Internet como soporte y el ciberespacio como lugar de socialización. Esas relaciones no son sólo de tipo lúdico, como aparecían al inicio alrededor de los MUD o juegos on line. Las comunidades virtuales se presentan, también, como un nuevo lugar para la formación del saber; un nuevo espacio antropológico donde compartir conocimientos y realizar aprendizajes.

\section{EL LUGAR DE LA INTELIGENCIA COLECTIVA}

Esta es la idea del pensador francés Pierre Lévy que propone, para nuestra época, un nuevo tipo de inteligencia que dirija esta nueva cultura y propone la creación de la inteligencia colectiva capaz de unir las aportaciones de todos sin excluir a nadie para afrontar los nuevos retos (Lévy, 1996: 34). Una inteligencia que está distribuida entre todos, que se coordina en tiempo real utilizando la Red y que aprovecha las competencias de cada individuo. 
En el origen de Internet, conviene recordarlo, estaba el deseo de compartir los conocimientos entre los grupos de científicos que trabajaban en el proyecto. Las primeras comunidades que se crearon en la Red, WELL, Fido Net, partían con el deseo de compartir informaciones, de dar la posibilidad a sus miembros de discutir sobre temas variados, dando la posibilidad, a cada persona, de ofrecer sus ideas en aquellos campos en los que es hábil (Rheingold, 1998: 151-163). Muchas de las comunidades virtuales que existen son, precisamente, lugares donde los miembros comparten sus conocimientos, trabajan conjuntamente, intercambian informaciones e iniciativas, ponen en común las propias adquisiciones. Internet permite conectar todas esas inteligencias como nunca antes se había pensado, en un pensamiento colectivo, que no único, donde cada persona se convierte en fuente de saber para los otros ${ }^{6}$.

Para este autor con las nuevas tecnologías se crea una nueva forma de continuidad en el tiempo y en el espacio. Ya los medios de comunicación de masas han creado esa comunidad, esa continuidad, pero no han creado una continuidad de pensamiento activo y vivo, sino una red de transporte de información. Si con los mass media la comunicación del saber se realizaba de forma jerárquica y unidireccional, con el desarrollo de las nuevas tecnologías de comunicación en red, y la creación de las comunidades virtuales, la comunicación del saber está abierta a todos los miembros. Internet, y las otras formas de comunicación online, pueden servir para crear nuevos espacios de comunicación, sin sustituir a otros y sin priorizar la importancia del medio. "Porque, es necesario subrayarlo, el mundo virtual es sólo el soporte de procesos cognitivos, sociales y afectivos que se producen entre personas reales. Así como la escritura y el teléfono no han impedido a las personas continuar encontrándose en carne y hueso, los mundos virtuales de los intelectuales colectivos no pretenden sustituirse al contacto directo. Al contrario, deben consentir a las personas que lo desean, de localizarse unos a otros y de agrandar el círculo de las propias relaciones de amistad, profesionales o de otro tipo. El mundo virtual es, seguramente, el medio de la inteligencia colectiva, pero no es ni la sede exclusiva, ni el origen ni el último fin" (Lévy, 1996: 119).

Según la idea de Lévy, la nueva tecnología de la Red permite a los usuarios de todo el mundo convertirse en parte activa en la construcción de esa inteligencia global. Las comunidades virtuales, se convertirán en "una entidad colectiva mundial, estructuralmente anti-autoritaria y anti-jerárquica" (Ferri, 1999a: 99) que harán posible este proceso. Las comunidades existentes ahora en la Red son los primeros intentos, los primeros pasos. Pienso que sigue planteando un interrogante a esta visión tan positiva de Lévy el que en muchas zonas no sea posible acceder a Internet por falta de medios y de una alfabetización adecuada a las nuevas tecnologías. Hay que contar además con que, aun en el caso de conseguir un acceso generalizado, los individuos deseen participar en esta construcción colectiva.

Todavía el mismo autor es consciente de que estamos en el momento inicial de una nueva sociedad, de una nueva cultura y que se debe proyectar cómo vamos a utilizarlas y para qué nos van

\footnotetext{
- Para Lévy el intelctual colectivo "costruisce un pensiero transpersonale e continuo. Una cogitazione anonima, ma perpetuamente viva, alimentata da mille fonti, metamorfica", (Lévy, 1996: 115).
} 
a servir ${ }^{7}$ ¿ ¿Hacia dónde se irá caminando en la implantación de las nuevas tecnologías? Un objetivo debe ser la creación de comunidades en el ciberespacio que sirvan para dar valor a lo humano y potencien el crecimiento de las personas.

No todas las formas de agregación en la Red son iguales o tienen las mismas características u objetivos. Para comprender mejor el fenómeno de las comunidades virtuales es necesario hacer una clasificación de las mismas.

\section{CLASIFICACIÓN DE LAS COMUNIDADES VIRTUALES}

Los tipos de comunidades que se presentan a continuación ${ }^{8}$, tienen, como primer objetivo, mostrar la diversidad y amplitud del fenómeno. Pero, por otra parte, pueden ayudar para darse cuenta de los posibles usos de Internet para agrupar a las personas.

\section{Comunidades de debate y discusión en tiempo real}

Son las más visitadas. Nos referimos aquí a los canales de discusión a través de aplicaciones de chat o Irc. Estas aplicaciones permiten dialogar con todas las personas que en ese momento están en el mismo canal en tiempo real (just in time). La conversación es, generalmente, escrita, aunque ya las aplicaciones permiten conversaciones con voz e imágenes si se dispone de un micrófono y una webcam. Los canales de chat suelen estar agrupados por temas y la lista de argumentos es variadísima. Normalmente hay una sala general donde todos pueden ir escribiendo sus comentarios $y$, si se desea, se puede abrir una ventana para hablar en privado, uno a uno, con uno de los participantes. Algunos portales dan la posibilidad de abrir un canal privado de chat donde invitar a otros participantes.

El tipo de relación que se establece puede ser muy efímero; los chats son un espacio de socialización muy flexible donde cada uno entra y sale cuando quiere. Es curioso que, precisamente, se utilice el término sala-salón para indicar su fin: charlar, conocer a gente nueva, entrar en contacto con otros. Los participantes deben adoptar un apodo (nickname) para ser identificables, pero que lo pueden cambiar a su antojo. A la hora de presentarse ante los demás, cada participante puede dar los datos de sí que le parezca oportuno o, incluso, crear una identidad distinta, práctica que suele ser bastante frecuente. A diferencia de otras aplicaciones de comunicación, las conversaciones del chat no pueden ser copiadas y desaparecen cuando se cierra el programa, aunque ya existen algunos programas que lo permiten. Los contactos que se establecen en este tipo de

\footnotetext{
7 "La cultura di rete non è ancora consolidata, le sue potenzialità tecniche sono ancora allo stadio iniziale, la sua crescita non è ancora terminata. Si è ancora in tempo per riflettere collettivamente e tentare di dare forma al corso degli eventi. (...) ci sembra prioritario mettere in luce le grandi possibilità di civilizzazione legate all'emergere del multimedia" (Lévy, 1996: 15).

8 Tomo algunos tipos de comunidades según la clasificación que presenta Paolo Ferri (Ferri, 1999b: 66-73).
} 
comunidades, si sólo se quedan aquí, son muy precarios. Es cierto que algunas veces se establece un contacto mayor con algunos miembros y la relación continúa con el teléfono, e-mail, o encuentro real.

\section{Comunidades de socialización, información, discusión o juego}

Aprovechando muchas de las aplicaciones que ofrece Internet (chat, grupos de noticias, listas de correo, espacios para páginas personales, paneles de mensajes) se han ido creando este tipo de comunidades. Respecto a las anteriores, el tipo de relación es más estable. Para formar parte de ellas es necesario inscribirse, dar algunos datos personales, aceptar un código de conducta. Exigen, por parte de los usuarios, una mayor dedicación y constancia. Se pueden incluir en este tipo las comunidades creadas alrededor de juegos online (los clásicos MUD). Otras, se presentan como puntos de encuentro y socialización alternativos a la sociedad real. Un ejemplo puede ser $\mathrm{Pe}$ gacity (www.pegacity.it) que se presenta como una ciudad virtual, dividida en barrios según las afinidades de quienes participan, con su alcalde y consejo de vecinos. La comunidad ofrece a cada ciudadano un espacio para construir su "casa", su home page, donde recibe a los que quieran visitarle. Sigue el modelo de la, ya clásica, WELL.

Este tipo de comunidades está más estructurado y organizado. Las relaciones que se establecen son más duraderas y exigen una mayor implicación personal. Los que forman parte de ellas lo hacen conscientes de querer aprovechar las posibilidades de la Red para establecer relaciones, lazos más estrechos con personas de cualquier parte del mundo. También dentro de esta categoría se pueden situar las redes cívicas, una interesante experiencia de comunidad virtual en un territorio determinado. Son los casos de algunas ciudades (citemos el caso de la red cívica de Milán, Bolonia o Ámsterdam) que ya están conectando a los ciudadanos y que se presentan como una nueva forma de socialización, de lugar de encuentro, entre los que viven en un área concreta.

\section{Comunidades temáticas de investigación o acción política}

Utilizando las aplicaciones típicas de Internet señaladas en el tipo anterior, estas comunidades se caracterizan por aglutinar a sus miembros alrededor de un tema concreto o una actividad de investigación. Algunos de los portales más famosos (como el www.msn.es de Microsoft) ofrecen ya elencos de comunidades dirigidas a los distintos grupos de profesionales, por ejemplo. Pensemos, también, en el tipo de relación que se crea alrededor de un portal o un sitio web entre estudiosos de un mismo argumento. Un aspecto interesante que subrayar en este apartado es la posibilidad, en esas comunidades de estudio, de confrontarse con personas y situaciones de realidades lejanas a donde se vive.

Dentro de este grupo están las emergentes comunidades de acción política. Internet está sirviendo como medio de unión entre personas de ideologías afines. Pero no sólo como medio de ex- 
posición de ideas o debates, sino como un verdadero medio que aglutina y da espacio a la socialización entre quienes comparten las mismas ideas. Además, está sirviendo para coordinar acciones a escala internacional. Basta pensar en las redes que se han ido tejiendo alrededor de las protestas antiglobalización? Internet, unida al teléfono móvil, se ha demostrado elemento fundamental para dar cohesión a esos grupos.

Alrededor de las páginas de algunos de estos grupos, los usuarios han podido contactar, conocer programas, conocerse entre ellos, coordinarse, intercambiar ideas y opiniones. Pero no sólo este tipo de grupos, también muchas organizaciones no gubernamentales están creando verdaderas redes de comunicación y de colaboración para llevar adelante sus proyectos. No se limitan a darse a conocer, sino que utilizan la Red como lugar de encuentro, de formación y de intercambio.

\section{Comunidades de organizaciones e instituciones}

Podemos incluir en este tipo las comunidades que surgen paralelas a instituciones gubernativas, políticas o sociales. Internet sirve para crear redes internas al servicio de esa institución. Hasta ahora la mayor parte de las instituciones que mantienen sitios web aprovechan una mínima parte de las posibilidades de Internet: función de marketing hacia fuera y comunicaciones internas. Pero las potencialidades del uso de las nuevas tecnologías en esta perspectiva van mucho más allá de eso y cambiaría la forma de ser de esas instituciones.

Con la posibilidad de acceso restringido, a través de contraseña (password), el sitio de la institución o el grupo puede convertirse en una verdadera comunidad virtual que reúne a los miembros. Hacia dentro de la misma institución, se podría mejorar el sentido de pertenencia a la misma, la toma de decisiones en común, democratizar y fomentar la participación de los miembros de esa institución, desarrollar formas de trabajo colaborativo más eficaces, diversificar los momentos de formación y puesta al día, mayor facilidad para participar en proyectos de esa institución incluso en diversas partes del mundo. También hacia fuera puede hacerse más clara la implantación en el territorio, sea local o transnacional, darse a conocer, contactar con nuevas personas interesadas en lo que esa institución ofrece, etc.

Como se ha visto, los tipos de participación e implicación en las comunidades virtuales son muy diversos. Como son diferentes los objetivos y fines de las distintas comunidades. Lo que es cierto es que se está utilizando Internet para establecer relaciones interpersonales con otros individuos, más allá de los límites del espacio geográfico impuestos por la interacción cara a cara. Ahora bien, este tipo de comunidad plantea algunos problemas que nacen de la tecnología que se utiliza y del tipo de interacción que se establece entre los participantes.

\footnotetext{
9 Así Nodo50 (www.nodo50.org) que se presenta como un territorio virtual de contrainformación y acción política en Internet. O, también, www.antiglobalizacion.org.
} 


\section{CRÍTICAS A LAS COMUNIDADES VIRTUALES}

La existencia de comunidades virtuales como forma de socialización suscita interrogantes. Algunos aspectos que nacen de la naturaleza de las mismas y la manera como se producen los contactos entre las personas que participan, son vistos como límites e incluso como riesgos. Señalamos algunos.

\section{¿Aislamiento de los individuos?}

Sería exagerado pensar en la existencia de comunidades virtuales como opuestas a las comunidades reales o sustitutas de éstas. Más bien hay que verlas "como una nueva modalidad del actuar comunicativo, en grado de potenciar y tal vez hacer más abierto y democrático el sistema de las relaciones comunitarias, sociales e institucionales, dentro de las cuales cada uno de nosotros está inserto" (Ferri, 1999a: 79).

Las comunidades virtuales son una nueva forma de agregación que se superponen a las otras ya existentes, no con la idea de excluir o cancelar a las otras. Podrían darse formas de uso de Internet que degenerasen en adicción con posibilidad de problemas psicopatológicos. Ya existe una cierta práctica clínica en el ámbito de la psiquiatría, que trata trastornos producidos por la adicción a Internet. Algunas personas con rasgos obsesivos-compulsivos pueden estar más expuestas a síndromes de adicción a Internet al generar comportamientos en los cuales "el sujeto se refugia en la Red para no afrontar problemáticas existenciales" (Cantelmi - D'Andrea, 2000: 59). Nos referimos a personas que tienden al aislamiento en las relaciones sociales y tienen propensión a inhibirse en las relaciones interpersonales. Ciertamente es un riesgo, pero del que no están exentas otras realidades de la vida ordinaria y que hace pensar más en Internet como el desencadenante, la ocasión que ha llevado a que se manifieste una problemática de la personalidad que ya existía previamente fuera de la Red.

Junto a esta consideración, también es cierto que otros consideran que "las relaciones sociales basadas sobre una modalidad textual pueden ser gratificantes para algunas personas" (Griffiths, 2000: 106), y las relaciones online pueden ser psicológicamente positivas, en algunas circunstancias, porque no tienen en cuenta prejuicios de tipo físico. Las relaciones no se basan en un primer lugar sobre aspectos físicos, sino sobre lo que el otro puede compartir conmigo durante el diálogo.

La comunidad virtual sería un ámbito más de relación del individuo que se sirve de ella para diversos fines, especialmente para las relaciones, el ocio y el conocimiento. Sería reducir a caricatura la esencia de las comunidades virtuales, pensar en un individuo aislado, cuyo único interlocutor es un ordenador, su único contacto con el mundo exterior la pantalla de la computadora y sus únicas relaciones interpersonales las que encuentra en una sala de chat. Como señala Sherry Turkle (Turkle, 1997: 367), en las comunidades virtuales debe darse una permeabilidad entre esos dos ámbitos, el real y el virtual, y deben producirse encuentros físicos entre algunos de los miembros de esas comunidades para que el término comunidad pueda ser aplicado a los mundos sociales virtuales. 
La misma dinámica de las comunidades virtuales, cuando funcionan, exige estos encuentros fuera del mundo del ordenador. Incluso en las comunidades virtuales de socialización más baja, esta exigencia está presente. Es el caso de los canales de chat. En la medida en que se establecen contactos reiterados con las mismas personas, suele surgir el interés por encontrar, fuera del chat, a la otra persona. Esta es una práctica que va haciéndose frecuente, como hemos podido comprobar en nuestras charlas con otros internautas. Es más, están surgiendo tipos de reuniones (Kedadas en el argot) de los participantes en una sala de chat, que se reúnen para conocerse, compartir el tiempo libre, etc.

\section{¿Problema de identidad?}

Uno de los aspectos críticos que se señalan de las comunidades virtuales es su anonimato, 0 mejor dicho, la posibilidad de presentarse con la identidad que uno quiera construirse. Hay que tener en cuenta que no todas las formas de participación en las comunidades virtuales son iguales y que la forma de estar en ellas varía. No es lo mismo participar en un chat (http://chat.ya.com) en el que se entra y sale cuando se quiere y sólo se debe elegir un nick, que participar en el grupo de discusión sobre temas religiosos (es.news.grupos), en el que hay que suscribirse y, se dan datos personales y hay que solicitar a nuestro proveedor de servicios darnos de alta para recibir las noticias.

Pero, en líneas generales, es cierto que en Internet el individuo puede presentarse con una personalidad construida. Esto es más claro en los MUD, los juegos de rol, donde el usuario debe construirse una personalidad ficticia de varón, hembra, animal, personaje fantástico, para jugar con los otros usuarios en Red. Una chica puede presentarse como un guerrero y mantener esa personalidad durante el juego (que puede durar semanas o meses), asumiendo el lenguaje, actitudes, sentimientos de ese personaje. En los salones de chat, por citar otra variedad de interacción en Internet, al inicio de una conversación se suelen preguntar y ofrecer datos personales, descripciones físicas, lugar desde donde se conecta, etc. Muchos de estos datos suelen ser inventados aunque, también es cierto, cuando se mantiene una conversación varias veces comienzan a darse informaciones más personales y reales.

Los usuarios de Internet no sólo son autores de lo que escriben, de los mensajes que lanzan, sino que "son autores de sí mismos, construyendo nuevos yo a través de la interacción social virtual" (Caretti, 2000: 126). Este intercambio social anónimo permite jugar con la personalidad y el aspecto físico. Seguramente la proliferación de las cámaras web reducirá este aspecto, pero ahora también la apariencia física depende de la descripción que el individuo hace. Las consecuencias psicológicas de este jugar con la propia identidad deben ser tomadas en consideración. Internet, como ya hemos señalado, puede ser un riesgo o una posibilidad.

Para Sherry Turkle, la posibilidad de crear diversos tipos de personalidad puede ser un valor del virtual, en el sentido que puede ayudar a conocer los distintos aspectos de la personalidad. Para ella, cada uno de nosotros formamos parte de diversos grupos (familia, amigos, grupo de trabajo, etc.). En 
cada uno de ellos se muestra aspectos de nuestra identidad, se establece un tipo de relaciones, no se excluyen unos a otros, sino que se compenetran. El individuo es, a la vez, padre, amigo, jefe en una oficina, miembro de una comunidad de creyentes. El papel que desempeña en cada uno de esos ámbitos es distinto y exige una serie de comportamientos, de formas de actuar e interactuar con los otros, diversos; distintas facetas de una misma personalidad. Internet, para esta autora, es un laboratorio donde experimentar la experiencia de la construcción y de la reconstrucción del yo en un proceso que permite modelarnos y re-crearnos a nosotros mismos (Turkle, 1997: 267).

\section{¿Carácter efímero y precariedad de las relaciones?}

Otro de los rasgos que, para algunos, definen a las comunidades virtuales es el carácter efímero de las relaciones que se establecen a través de la Red ${ }^{10}$. Esta facilidad para establecer contactos, charlar y no volver a contactar con esa persona produciría una precariedad en las relaciones. Los contactos que en ellas se establecen son, para algunos ${ }^{11}$, superficiales pues esa inestabilidad en las relaciones es un elemento connatural a las comunidades virtuales. Las comunidades tradicionales que conocemos (partidos, instituciones, estados, etc.) se caracterizan, en este sentido, por la solidez y estabilidad de las relaciones entre sus miembros; son comunidades estructuradas en las que existe un acuerdo implícito de estabilidad en las relaciones de pertenencia de sus miembros.

En las comunidades virtuales es la provisionalidad el elemento estructural de las mismas y están destinadas a disolverse. Un tipo de comunidad así, se piensa, no es capaz de dar sentido, de influenciar la realidad social de las personas, de crear unas relaciones sociales densas, sino que fragmentan el sentido de pertenencia de los individuos y debilitan los lazos que estos mantienen con las comunidades tradicionales. Se señala el carácter provisional de muchas de las relaciones que se establecen en estas comunidades y los límites de las relaciones que pueden establecerse dentro de ellas.

Pero, también podría pensarse que esas notas (fragilidad, fragmentación, debilidad en las relaciones) no son características intrínsecas de las comunidades virtuales, sino reflejo de las formas de relación que se dan en nuestra sociedad hoy (Ferri, 1999b: 88), en un momento en que se están repensando el tipo de relaciones entre los individuos y entre los individuos y las instituciones. Es posible que las comunidades virtuales se estén convirtiendo en lugares donde explorar nuevas formas de socialización, "vehículos de nuevas agregaciones sociales" (Ferri, 1999b: 89); un nuevo contexto social y comunitario que no se opone a las otras formas de agregación, sino que coexiste con ellas ampliándolas y completándolas.

\footnotetext{
10 Una exposición de diversas posturas críticas a este respecto en Ferri (Ferri, 1999a: 84-91).

1 "ll ciberspazio diviene spesso il palcoscenico per creare delle indentità fittizie con cui soggetti deboli si divertono a chiacchierare e a giocare, generando però delle comunità spesso instabili e fittizie", CIPOLLA - Dell'AQUILA, Cibernauti di tutta Italia unitevi in comunità, virtuali.
} 
En un mundo globalizado, las nuevas tecnologías de la comunicación ofrecen la posibilidad de hacer real un tipo de socialización también global, que pone en contacto a personas eliminando las distancias físicas. También puede verse, detrás de este fenómeno, un deseo de los hombres y mujeres de nuestro tiempo de comunicarse, la necesidad del encuentro con el otro y de compartir experiencias y sentimientos.

\section{NUEVOS TIPOS DE SOCIALIZACIÓN}

Teniendo presente los anteriores aspectos críticos de este tipo de comunidades, conviene señalar que estamos ante un nuevo tipo de socialización. Este tipo de relaciones se suman a las que ya practica el individuo y se añaden a las ya existentes. No parece que el uso de Internet para establecer relaciones sociales "conduzca a una menor interacción y a un mayor aislamiento social"(Castells, 2001: 145). Sin olvidar el dato importante de que muchas de las comunidades virtuales son prolongación de los grupos ya existentes en el mundo físico. Y que numerosas comunidades online buscan una prolongación offline entre los componentes. Se está produciendo una mezcla de las dos realidades. El mundo virtual influye en el físico y se produce una superposición de relaciones sociales.

Hay que hacer notar, además, que son distintos los niveles de participación en las comunidades virtuales. Hay que tenerlos en cuenta para valorarlas y para pensar los contenidos de las mismas y las funciones que pueden desarrollar. Existen diversos grados de participación en las comunidades virtuales. Esta participación puede ir desde formas más superficiales de relación, como puede ser la intervención esporádica en una sala de chat, a formas más estables de pertenencia. En este último caso se exige un alto grado de participación y de empeño personal. Quien entra para divertirse solamente se cansa y abandona al poco tiempo. Esto puede observarse entre los participantes de un chat o de un foro de discusión: se constatan algunos nombres y nicks que se repiten con frecuencia, y otros que aparecen sólo alguna vez. Cuando se consolida la participación de los individuos en una comunidad pueden darse formas interesantes y profundas de relación interpersonal.

Participar en comunidades virtuales es algo más que utilizar Internet para visitar páginas web, algo más que surfear por la Red. Quien visita una página web, normalmente, está buscando información o materiales que puedan serle útil para una actividad concreta. En cambio la persona que participa en un foro de discusión o un canal Irc, lo que está buscando es entrar en contacto con otras personas que comparten sus mismas inquietudes, discutir sobre temas que le interesan, aumentar sus conocimientos sobre algunos temas, o simplemente charlar con otros individuos e intercambiarse experiencias. El tipo de actitud que se requiere es diverso; hace falta una actitud activa pues la comunidad se construye entre todos.

\section{REFERENCIAS BIBLIOGRÁFICAS}

- Cantelmi, T.; Talli, M. (eds.) (2000): DEL MIGLIO C.- D'ANDREA A., La mente in Internet. Psicopatologia delle condotte on-line. Padua: Piccin. 
- Carbone, P.; Ferri, P. (eds) (1999): Le comunità virtuali, Milán: Mimesis.

- Castells, M. (2001): La galaxia Internet. Reflexiones sobre Internet, empresa y sociedad, Madrid: Plaza \& Janés.

- Cipolla, C.; Dell'Aquila, P. (1998): "Cibernauti di tutta Italia unitevi in comunità, virtuali", Telèma, vol. 13.

- Fabietti, U. (1999): "Comunità dense, comunità immaginate, comunità virtuali. Un pundo di vista antropologico", en Carbone, P. ; Ferri, P. (eds) (1999): Le comunità virtuali. Milán: Mimesis, pp. 43-61.

- Ferri, P. (1999a): La rivoluzione digitale. Comunità, individuo e testo nell'era di Internet, Milán: Mimesis.

- Ferri, P. (1999b): "Comunità e comunità virtuale; due concetti a confronto", en Carbone, P. ; Ferri, P., (eds) (1999): Le comunità virtuali, Milán: Mimesis, pp. 79-105.

- Holenton, R. (1998): Composing cyberspace. Identity, community and knowledge in the electronic age, Boston: McGraw Hill.

- Lévy, P. (1996): L'intelligenza collettiva. Per un'antropologia del cyberspazio, Milán: Feltrinelli.

- Lévy, P. (1997): II virtuale, Milán: Raffaello Cortina.

- Lévy, P. (2001): Cybercultura. Gli usi sociali delle nuove tecnologie, Milán: Feltrinelli.

- Maldonado, T. (1997): Critica della ragione informatica, Milán: Feltrinelli.

- Rheingold, H. (1994): Comunità virtuali. Parlare, incontrarsi, vivere nel cyberspazio, Milán: Sperling \& Kupfer.

- Turkle, S. (1997): La vita sullo schermo. Nuove identità e relazioni sociali nell'epoca di Internet, Milán: Apogeo. 